Pacific Journal of Mathematics

LONG WALKS IN THE PLANE WITH FEW COLLINEAR 


\title{
LONG WALKS IN THE PLANE WITH FEW COLLINEAR POINTS
}

\author{
JoSeph L. GeRVer
}

Let $S$ be a set of vectors in $R^{n}$. An $S$-walk is any (finite or infinite) sequence $\left\{z_{i}\right\}$ of vectors in $\boldsymbol{R}^{\boldsymbol{n}}$ such that $z_{i+1}-z_{i} \in S$ for all $i$. We will show that if the elements of $S$ do not all lie on the same line through the origin, then for each integer $K \geqq 2$, there exists an $S$-walk $W_{K}=\left\{z_{i}\right\}_{i=1}^{N(K)}$ such that no $K+1$ elements of $W_{K}$ are collinear and $N(K)$ grows faster than any polynomial function of $K$.

Specifically, we will prove that

$$
\log _{2} N(K)>\frac{1}{9}\left(\log _{2} K-1\right)^{2}-\frac{1}{6}\left(\log _{2} K-1\right) .
$$

We will then show that if the elements of $S$ lie on at least $L$ distinct lines through the origin, then there exists an $S$-walk of length $N(K, L)$ with no $K+1$ elements collinear, such that $N(K, L) \geqq$ $(1 / 4) L^{*} N(K-1)$, where $L-2 \leqq L * \leqq L+1$ and $L * \equiv 0 \bmod 4$. In [3] it was shown that if $S \subset Z^{2}$, and for all $s \in S$ we have $\|s\| \leqq M$, then there does not exist an $S$-walk $W=\left\{z_{i}\right\}_{i=1}^{N(K, M)}$ such that no $K+1$ elements of $W$ are collinear and

$$
\log _{2} N(K, M)>2{ }^{13} M^{4} K^{4}+\log _{2} K \text {. }
$$

Before proving these theorems we introduce some notation. If $A=\left(\boldsymbol{a}_{1}, \cdots, \boldsymbol{a}_{n}\right)$ and $B=\left(\boldsymbol{b}_{1}, \cdots, \boldsymbol{b}_{m}\right)$ are ordered sets of vectors, we let $R A=\left(\boldsymbol{a}_{n}, \cdots, \boldsymbol{a}_{1}\right)$ and we let $(A, B)=\left(\boldsymbol{a}_{1}, \cdots, \boldsymbol{a}_{n}, \boldsymbol{b}_{1}, \cdots, \boldsymbol{b}_{m}\right)$. We let $2 A=(A, A)$ and, for every positive integer $k$, we let $(k+1) A=$ $(k A, A)$. If $J$ is a vector operator, we let $J A=\left(J \boldsymbol{a}_{1}, \cdots, J \boldsymbol{a}_{n}\right)$.

THEOREM 1. Let $S$ contain two vectors independent over $\boldsymbol{R}$, and let $K$ be an integer greater than or exual to 2. There exists an S-walk $W_{K}=\left\{z_{p}\right\}_{p=1}^{N(K)}$ such that no $K+1$ elements of $W_{K}$ are collinear and such that

$$
\log _{2} N(K)>\frac{1}{9}\left(\log _{2} K-1\right)^{2}-\frac{1}{6}\left(\log _{2} K-1\right) .
$$

Proof. If we let $\left(\log _{2} K-1\right)^{2} / 9-\left(\log _{2} K-1\right) / 6=\log _{2} K$, then $\log _{2} K=(25+3 \sqrt{65}) / 4>12$ or $(25-3 \sqrt{65}) / 4<1$. Therefore if $1 \leqq \log _{2} K \leqq 12$, and $2 \leqq K \leqq 4096$, then 


$$
\frac{1}{9}\left(\log _{2} \mathrm{~K}-1\right)^{2}-\frac{1}{6}\left(\log _{2} K-1\right)<\log _{2} K .
$$

Since $W_{K}$ cannot have more than $N(K)$ collinear points, we need only consider $K>4096$.

We may let $S=\{i, j\}$ without loss of generality, where $i$ and $j$ are orthonormal unit vectors.

For every positive integer $m$ and nonnegative integer $n$, let $A_{0}^{m}=i$, and let

$$
A_{n+1}^{m}=\left(m A_{n}^{m}, 2^{n} R J A_{n}^{m}\right),
$$

where $J i=j$ and $J j=i$. Let $V=\left\{\boldsymbol{v}_{p}\right\}_{p=1}^{N}=\mu A_{\nu}^{\mu}$, where $\mu$ is the greatest integer less than or equal to $((7 / 9) K)^{1 / 3}$, and $\nu$ is the least integer greater than or equal to $\log _{2} \mu-3 / 2$. Note that since $K>$ 4096, we have $\mu \geqq 14$, and $\nu \geqq 3$. Let $z_{p}=\sum_{q=1}^{p} \boldsymbol{v}_{q}$ for each $p$, and let $W=\left\{z_{p}\right\}_{p=1}^{N}$. We maintain that $W$ has no more than $K$ collinear points and that $\log _{2} N>\left(\log _{2} K-1\right)^{2} / 9-\left(\log _{2} K-1\right) / 6$.

Let $b_{0}=1$ and let $b_{n+1}=\left(\mu+2^{n}\right) b_{n}$. Then $b_{n}$ is the cardinality of $A_{n}^{\mu}$, and $N=\mu b_{\nu}$. Clearly $b_{n} \geqq \mu^{n}$, so $N \geqq \mu^{\nu+1}$ and $\log _{2} N \geqq$ $(\nu+1) \log _{2} \mu \geqq\left(\log _{2} \mu-1 / 2\right) \log _{2} \mu$. Since $\mu$ is the greatest integer less than or equal to $((7 / 9) K)^{1 / 3}$, and $((7 / 9) K)^{1 / 3}>14$, we have $\mu>$ $(14 / 15)((7 / 9) K)^{1 / 3}>((1 / 2) K)^{1 / 3}$. It follows that $\log _{2} N>1 / 9\left[\log _{2}((1 / 2) K)\right]^{2}-$ $\log _{2}((1 / 2) K) / 6=\left(\log _{2} \mathrm{~K}-1\right)^{2} / 9-\left(\log _{2} K-1\right) / 6$.

We now prove that $W$ has no more than $K$ collinear points.

Let $C_{n}^{\alpha}=\left\{z_{p}: \alpha b_{n} \leqq p \leqq(\alpha+1) b_{n}\right\}$. For each $n$, all $C_{n}^{\alpha}$ are congruent; specifically one can get from any one to any other by a translation plus, possibly, a reflection about the major diagonal (i.e., a reflection about the line passing through the vector $i+j$, which interchanges $i$ and $j$ ), followed by a rotation about the origin of $180^{\circ}$. This reflection plus rotation is equivalent to a reflection about the line perpendicular to the major diagonal (i.e., the line passing through the vector $i-j)$. We will refer to this latter line as the minor diagonal. Let

$$
\begin{gathered}
U_{n}^{\beta}=\left\{C_{n}^{\alpha}: \beta\left(\mu+2^{n}\right) \leqq \alpha<(\beta+1)\left(\mu+2^{n}\right)\right. \\
\text { if } n \neq \nu \text { and } U_{\nu}^{0}=\left\{C_{\nu}^{\alpha}: 0 \leqq \alpha \leqq \mu\right\}
\end{gathered}
$$

Note that $C_{n+1}^{\beta}=\left\{z_{p}: \beta\left(\mu+2^{n}\right) b_{n} \leqq p \leqq(\beta+1)\left(\mu+2^{n}\right) b_{n}\right\}$, so $U_{n}^{\beta}$ is a partition of $C_{n+1}^{\beta}$ and $U_{\nu}^{0}$ is a partition of $W$. We now consider a line with slope $m$ and determine for each $n$, the maximum number of elements of $U_{n}^{\beta}$ which the line can intersect (the maximum number cannot depend on $\beta$, since all $C_{n+1}^{\beta}$ are congruent). Let $r_{n}$ be this maximum number. Then the line cannot intersect more than $r=$ $\prod_{n=0}^{\nu} r_{n}$ points of $W$. 
Let $s_{n}$ be the slope of $z_{b_{n}}$; i.e., $s_{n}=y_{n} / x_{n}$ where $z_{b_{n}}=x_{n} i+y_{n} \boldsymbol{j}$. The slope of $z_{(\alpha+1) b_{n}}-z_{\alpha b_{n}}$ is then either $s_{n}$ or $s_{n}^{-1}$, depending on whether $C_{n}^{\alpha}$ is a simple translation of $C_{n}^{0}$, or a translation of the reflection of $C_{n}^{0}$ about the minor diagonal. We wish to find a lower bound on $s_{n} / s_{n-1}$.

Now $x_{0}=1, y_{0}=0, x_{n+1}=\mu x_{n}+2^{n} y_{n}$, and $y_{n+1}=\mu y_{n}+2^{n} x_{n}$. It follows that $x_{n}, y_{n}$, and $s_{n}$ are strictly positive for all $n \geqq 1$. We now prove by induction that $s_{n}<2^{n} / \mu$. Clearly $s_{0}=0<2^{0} / \mu$ and $s_{1}=1 / \mu<2^{1} / \mu$. Suppose $s_{n}<2^{n} / \mu$. Let $t_{n}=2^{n} / s_{n} \mu$. Then $t_{n}>1$. Now

$$
\begin{aligned}
s_{n+1} & =\left(\mu y_{n}+2^{n} x_{n}\right) /\left(\mu x_{n}+2^{n} y_{n}\right) \\
& =\left(\mu s_{n}+2^{n}\right) /\left(\mu+2^{n} s_{n}\right) \\
& =\left(\mu s_{n}+\mu s_{n} t_{n}\right) /\left(\mu+\mu s_{n}^{2} t_{n}\right) \\
& =\left(s_{n}+s_{n} t_{n}\right) /\left(1+s_{n}^{2} t_{n}\right) .
\end{aligned}
$$

Thus

$$
\begin{aligned}
t_{n+1} & =2^{n+1} / s_{n+1} \mu=2 s_{n} t_{n} / s_{n+1} \\
& =2 s_{n} t_{n}\left(1+s_{n}^{2} t_{n}\right) /\left(s_{n}+s_{n} t_{n}\right) \\
& =2 t_{n}\left(1+s_{n}^{2} t_{n}\right) /\left(t_{n}+1\right) .
\end{aligned}
$$

We now view $t_{n+1}$ as a function of the real variables $t_{n}$ and $s_{n}$, and compute its partial derivatives:

$$
\partial t_{n+1} / \partial t_{n}=2\left(s_{n}^{2} t_{n}^{2}+2 s_{n}^{2} t_{n}+1\right) /\left(t_{n}+1\right)>0
$$

and

$$
\partial t_{n+1} / \partial s_{n}=4 t_{n}^{2} s_{n} /\left(t_{n}+1\right)>0 .
$$

Since $t_{n+1}$ has the value 1 when $s_{n}=0$ and $t_{n}=1$, it follows that $t_{n+1}>1$ when $s_{n} \geqq 0$ and $t_{n}>1$, as is the case here. Therefore $s_{n+1}<2^{n+1} / \mu$.

Next, recall that $\nu-1<\log _{2} \mu-3 / 2$, so if $n \leqq \nu-1$, then $2^{n} \leqq 2^{\nu-1}<2^{-3 / 2} \mu$. Since $2^{n}>s_{n} \mu$, it follows firstly that $s_{n}<2^{-3 / 2}$, and secondly that

$$
\begin{aligned}
s_{n+1} / s_{n} & =\left(\mu s_{n}+2^{n}\right) /\left(\mu s_{n}+2^{n} s_{n}^{2}\right) \\
& >2 \mu s_{n} /\left(\mu s_{n}+2^{-3 / 2} \mu s_{n}^{2}\right) \\
& =2 /\left(1+2^{-3 / 2} s_{n}\right)>2 /\left(1+\frac{1}{8}\right)=\frac{16}{9} .
\end{aligned}
$$

It follows that, given $m$, there is at most one $n$ such that $(3 / 4) s_{n} \leqq m \leqq(4 / 3) s_{n}$. Suppose there exists $\lambda$ such that $(3 / 4) s_{\lambda} \leqq m \leqq$ $(4 / 3) s_{\lambda}$. Then $m<(3 / 4) s_{\lambda+1}$ and $m>(4 / 3) s_{\lambda-1}$. Moreover, for all $n>$ $\lambda+1$, we have $m<(27 / 64) s_{n}<(1 / 2) s_{n}$, and for all $n<\lambda-1$, we 
have $m>(64 / 27) s_{n}>2 s_{n}$. All of the above also holds if we replace $s_{n}$ by $s_{n}^{-1}$, except that some of the inequalities are reversed and constants replaced by their reciprocals in the obvious way.

We now calculate for each of the five cases, $n=\lambda, n=\lambda+1$, $n=\lambda-1, n>\lambda+1$, and $n<\lambda-1$, the maximum number $r_{n}$ of elements of $U_{n}^{\beta}$ which a line of slope $m$ can intersect. We can assume without loss of generality that $C_{n+1}^{\beta}$ is a simple translation of $C_{n+1}^{0}$; if $C_{n+1}^{\beta}$ is a translation of the reflection of $C_{n+1}^{0}$ about the minor diagonal, then we can apply the same argument, replacing $s_{n}$ by $s_{n}^{-1}$. Then $C_{n}^{\alpha}$ is a simple translation of $C_{n}^{0}$ for $\beta\left(\mu+2^{n}\right) \leqq$ $\alpha<\beta\left(\mu+2^{n}\right)+\mu$, and a translation of the reflection of $C_{n}^{0}$ for $\beta\left(\mu+2^{n}\right)+\mu \leqq \alpha<(\beta+1)\left(\mu+2^{n}\right)$. For each $\alpha$, the first point of $C_{n}^{\alpha+1}$ coincides with the last point of $C_{n}^{\alpha}$. It is easy to prove by induction on $n$ that $C_{n}^{0}$ (and therefore $C_{n}^{\alpha}$ for all $\alpha$ ) lies entirely within a right triangle, with sides $x_{n}$ and $y_{n}$ adjacent to the right angle, and with the first and last points of $C_{n}^{0}$ at opposite ends of the hypotenuse. Therefore the sets $C_{n}^{\alpha}: \beta\left(\mu+2^{n}\right) \leqq \alpha<\beta\left(\mu+2^{n}\right)+$ $\mu$ lie within congruent right triangles, whose hypotenuses are adjacent segments of a line with slope $s_{n}$ (see Fig. 1). It follows

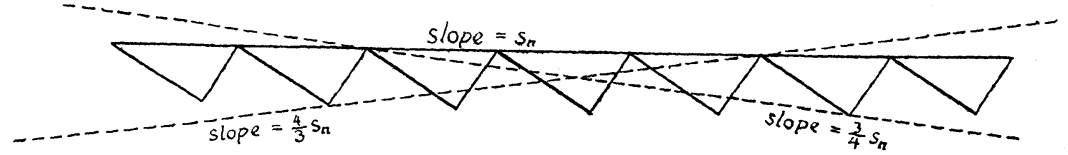

Figure 1

that a line with slope $m>s_{n} q /(q-1)$ or $m<s_{n}(q-1) / q$ can intersect at most $q$ of the sets $C_{n}^{\alpha}: \beta\left(\mu+2^{n}\right) \leqq \alpha<\beta\left(\mu+2^{n}\right)+\mu$ at distinct points (i.e., assign the last point of each set $C_{n}^{\alpha}$ to the set $C_{n}^{\alpha+1}$, and do not count the line as intersecting $C_{n}^{\alpha}$ if it only intersects this last point). Suppose $m \leqq 1$. Then $m<(1 / 2) s_{s}^{-1}$, and a line of slope $m$ can intersect no more than two of the sets $C_{n}^{\alpha}: \beta\left(\mu+2^{n}\right)+$ $\mu \leqq \alpha<(\beta+1)\left(\mu+2^{n}\right)$. If $n=\lambda$, then a line of slope $m$ can intersect all $\mu$ of the sets $C_{n}^{\alpha}: \beta\left(\mu+2^{n}\right) \leqq \alpha<\beta\left(\mu+2^{n}\right)+\mu$ for a total of $\mu+2$. If $n=\lambda+1$ or $\lambda-1$, the line can intersect at most 4 of the sets $C_{n}^{\alpha}: \beta\left(\mu+2^{n}\right) \leqq \alpha<\beta\left(\mu+2^{n}\right)+\mu$, for a total of 6 , while if $n>\lambda+1$ or $n<\lambda-1$, the line can intersect at most two of the sets $C_{n}^{\alpha}: \beta\left(\mu+2^{n}\right) \leqq \alpha<\beta\left(\mu+2^{n}\right)+\mu$ for a total of 4. If $m>1$, then we obtain essentially the same results by redefining $\lambda$ so that $(3 / 4) s_{\lambda}^{-1} \leqq m \leqq(4 / 3) s_{\lambda}^{-1}$, the only difference being that $\mu$ is replaced by $2^{n}$, which in any case is less than $\mu$. Therefore we have $r_{n} \leqq \mu+2$ if $n=\lambda, r_{n} \leqq 6$ if $n=\lambda-1$ or $\lambda+1$, and $r_{n} \leqq 4$ for all other $n$. Finally, we have 


$$
\begin{aligned}
r & =\prod_{n=0}^{\nu} r_{n} \leqq(\mu+2) \cdot 6^{2} \cdot 4^{\nu-2}<36(\mu+2) \cdot 4^{\log _{2 \mu-5 / 2}} \\
& =\frac{36}{32} \mu^{2}(\mu+2) \leqq \frac{9}{7} \mu^{3} \leqq K
\end{aligned}
$$

If $\lambda$ does not exist, then there are at most two values of $n$ for which $(27 / 64) s_{n} \leqq m \leqq(64 / 27) s_{n}$, and these two values can take the place of $\lambda-1$ and $\lambda+1$ in our argument.

REMARK. We can use this method to get slightly better results as follows: The method works by partitioning $W$ into a heiarchy of sets, each set of order $n+1$ being partitioned into $\mu+2^{n}$ sets of order $n$, and showing that for almost all $n$, a given line can intersect at most four sets of order $n$ within a given set of order $n+1$. Suppose that instead of using the partition based on the sets $C_{n}^{\alpha}$, we modify this partition slightly by splitting each $C_{n}^{\alpha}$ into two sets of order $n$, namely $\left\{z_{p}: \alpha b_{n} \leqq p \leqq \alpha b_{n}+\mu b_{n-1}\right\}$ and $\left\{z_{p}: \alpha b_{n}+\right.$ $\left.\mu b_{n-1} \leqq p \leqq(\alpha+1) b_{n}\right\}$. Then each set of order $n+1$ would have either $2 \mu$ or $2^{n+1}$ sets of order $n$, and it should not be hard to show that for almost all $n$, a given line can intersect at most three sets of order $n$ within a given set of order $n+1$. We would then have $r=c \mu \cdot 3^{\nu}=c \mu^{1+\log _{2} 3}$, where $c$ is a constant which does not depend on $K$, and finally

$$
\log _{2} N=\left(1+\log _{2} 3\right)^{-2}\left(\log _{2} K\right)^{2}+O\left(\log _{2} K\right) .
$$

However, it seems impossible to push this method any further.

THeOREM 2. Suppose that $S$ contains $L$ elements which are pairwise independent over $\boldsymbol{R}$. Then there exists an $S$-walk $\Omega=$ $\left\{\boldsymbol{u}_{i}\right\}_{i=1}^{N}$ containing no set of $K+1$ collinear points, such that

$$
\log _{2} N>\frac{1}{9}\left[\log _{2}(K-1)-1\right]^{2}-\frac{1}{6}\left[\log _{2}(K-1)-1\right]+\log _{2} L^{*}-2,
$$

where $L-2 \leqq L^{*} \leqq L+1$ and $L^{*} \equiv 0 \bmod 4$.

Proof. The $L$ elements of $S$ with distinct arguments must include $L / 2$ elements (if $L$ is even) or $(L+1) / 2$ elements (if $L$ is odd) in the same half-plane. Label these elements $\boldsymbol{s}_{1}, \boldsymbol{s}_{2}, \boldsymbol{s}_{3}, \ldots$ in order of their arguments. For $1 \leqq n \leqq(1 / 4) L^{*}$, let $W_{n}=\varphi_{n} W$ where $W$ is defined as in the proof of Theorem 1 , and $\varphi_{n}$ is the linear vector operator which maps $i$ to $\boldsymbol{s}_{2 n-1}$ and $\boldsymbol{j}$ to $\boldsymbol{s}_{2 n}$. Let $N_{0}$ be the cardinality of $W$ and let $\boldsymbol{w}_{n}=x \boldsymbol{s}_{2 n-1}+y \boldsymbol{s}_{2 n}$ be the final element of $W_{n}$. For $1 \leqq i \leqq N_{0}$, let $z_{i}$ be defined as in the proof of Theorem 1, and let $u_{i}=\varphi_{1} z_{1}$. Let $u_{N_{0} n+i}=\sum_{j=1}^{n} w_{j}+\varphi_{n+1} z_{i}$ for 
$1 \leqq n \leqq(1 / 4) L^{*}-1$. Finally, let $N=(1 / 4) L^{*} N_{0}$ and let $\Omega=\left\{\boldsymbol{u}_{i}\right\}_{i=1}^{N}$. Note that $\Omega$ is constructed by placing the $W_{n}$ end to end in sequence.

By Theorem 1,

$$
\log _{2} N>\frac{1}{9}\left(\log _{2} K-1\right)^{2}-\frac{1}{6}\left(\log _{2} K-1\right)+\log _{2} L^{*}-2 .
$$

We will now prove that no $K+2$ points of $\Omega$ are collinear. Substituting $K-1$ for the bound variable $K$ then gives us Theorem 2 for the case $K \geqq 3$. For the case $K=2$, we simply let $\boldsymbol{u}_{i}=$ $\sum_{j=1}^{i} \boldsymbol{s}_{j}$. The resulting set $\left\{\boldsymbol{u}_{i}\right\}$, which contains at least $(1 / 2) L^{*}$ elements, is the set of vertices of a convex polygon; hence no three elements are collinear.

Let $T_{n}=\left\{u_{i}\right\}_{i=N_{0}(n-1)+1}^{N_{0} n}$ and let $\boldsymbol{t}_{n}=\sum_{j=1}^{n} \boldsymbol{w}_{j}$, so that $\boldsymbol{t}_{n}$ is the final element of $T_{n}$. Let $t_{0}=0$ and let $r_{n}=t_{n-1}+x s_{2 n-1}$ for $n \geqq 1$. Note that $t_{n}=r_{n}+y s_{2 n}$. Note also that from results proved previously, the set $T_{n}$ must lie entirely on or in the interior of the triangle $\Delta_{n}$ with vertices $\boldsymbol{t}_{n-1}, \boldsymbol{r}_{n}$, and $\boldsymbol{t}_{n}$. Consequently any line which intersects $T_{n}$ must intersect $A_{n}$. Now consider the polygon $P$ with vertices $t_{0}, r_{1}, t_{1}, r_{2}, t_{2}, \cdots, r_{L^{*} / 4}, t_{L^{* / 4}}$ in that order. The (directed) edges of this polygon are the vectors $x \boldsymbol{s}_{1}, y \boldsymbol{s}_{2}, x \boldsymbol{s}_{3}, \cdots$, $y \boldsymbol{s}_{L^{*} / 2}$, and $-x \sum_{n=1}^{I * / 4} \boldsymbol{s}_{2 n-1}-y \sum_{n=1}^{L^{*} / 4} \boldsymbol{s}_{2 n}$. Since the vectors $\boldsymbol{s}_{1}, \boldsymbol{s}_{2}, \boldsymbol{s}_{3}, \cdots$ are listed in order of increasing argument, and the range of all their arguments is less than $180^{\circ}$, it follows that the interior angles of $P$ are all less than $180^{\circ}$, so $P$ is convex. Now any line intersecting $\Delta_{n}$, and in particular any line intersecting $T_{n}$, must intersect at least two sides of $\Delta_{n}$ (including each vertex in its two adjacent sides), and therefore must intersect $P$. Since $P$ is convex, a line can only intersect $P$ at one or two points, or along an edge. Therefore no line can intersect more than two of the $T_{n}$. Unless the slope of a line is between that of $\boldsymbol{s}_{2 n-1}$ and $\boldsymbol{s}_{2 n}$ inclusive, it can only intersect one point of $T_{n}$. By Theorem 1, no line can intersect more than $K$ points of $T_{n}$. Therefore, no line can contain more than $K+1$ points of $\Omega$.

REMARK. In order to compare these results with the upper bound in [3], we can consider the case where $S=\left\{s \in Z^{2}:\|s\| \leqq M\right\}$. Since the number of lattice points in a disc of radius $R$ is $\pi R^{2}+$ $O(R)$ [2], we know that the number of lattice points with both coordinates divisible by $q$, in a disc of radius $M$, is $\pi M^{2} / q^{2}+O(M / q)$. Therefore the number $L$ of lattice points with relatively prime coordinates is

$$
\pi M^{2} \sum_{n=0}^{\infty}(-1)^{n} \sum_{q \in Q_{n}} q^{-2}+O\left(M \sum_{q \in Q} q^{-1}\right),
$$


where $Q$ is the set of square free positive integers less than or equal to $M$, and $Q_{n}$ is the set of integers in $Q$ with $n$ distinct prime factors. It follows [1] that

$$
L=6 M^{2} / \pi+O(M \log M) .
$$

Finally, if we let $N(K, M)$ be the length of the longest $S$-walk with no more than $K$ collinear points, and we choose any constants $c_{1}<(9 \log 2)^{-1}$ and $c_{2}>2^{13} \log 2$, then we have

$$
M^{2} \exp \left[c_{1}(\log K)^{2}\right]<N(K, M)<\exp \left[c_{2} M^{4} K^{4}\right]
$$

for all $M$ and all but a finite number of $K$.

\section{REFERENCES}

1. T. M. Apostol, Introduction to Analytic Number Theory, Springer-Verlag, New York, (1976), 63.

2. H. Rademacher, Lectures on Elementary Number Theory, Blaisdell, New York, (1964), 100.

3. L. T. Ramsey and J. L. Gerver, On certain sequences of lattice points, Pacific J. Math., 83 (1979), 357-363.

Received June 16, 1978.

UNIVERSITY OF HAWAII

HoNOLULU, HI 96822

Current address: UNIVERSITY OF GEORGIA

Athens, GA 30602 



\title{
PACIFIC JOURNAL OF MATHEMATICS
}

\section{EDITORS}

DONALD BABBITT (Managing Editor)

University of California

Los Angeles, CA 90024

\section{HUGO RossI}

University of Utah

Salt Lake City, UT 84112

C. C. MOORE and ANDREW OGG

University of California

Berkeley, CA 94720

\section{J. DUGUNDJI}

Department of Mathematics University of Southern California Los Angeles, CA 90007

R. FINN and J. Milgram

Stanford University Stanford, CA 94305

\section{ASSOCIATE EDITORS}
E. F. BECKENBACH
B. H. NeUmanN
F. WOLF
K. YosHIDA

\section{SUPPORTING INSTITUTIONS}

\author{
UNIVERSITY OF BRITISH COLUMBIA \\ CALIFORNIA INSTITUTE OF TECHNOLOGY \\ UNIVERSITY OF CALIFORNIA \\ MONTANA STATE UNIVERSITY \\ UNIVERSITY OF NEVADA, RENO \\ NEW MEXICO STATE UNIVERSITY \\ OREGON STATE UNIVERSITY \\ UNIVERSITY OF OREGON
}

\author{
UNIVERSITY OF SOUTHERN CALIFORNIA \\ STANFORD UNIVERSITY \\ UNIVERSITY OF HAWAII \\ UNIVERSITY OF TOKYO \\ UNIVERSITY OF UTAH \\ WASHINGTON STATE UNIVERSITY \\ UNIVERSITY OF WASHINGTON
}

The Supporting Institutions listed above contribute to the cost of publication of this Journal, but they are not owners or publishers and have no responsibility for its content or policies.

Mathematical papers intended for publication in the Pacific Journal of Mathematics should be in typed form or offset-reproduced, (not dittoed), double spaced with large margins. Please do not use built up fractions in the text of the manuscript. However, you may use them in the displayed equations. Underline Greek letters in red, German in green, and script in blue. The first paragraph or two must be capable of being used separately as a synopsis of the entire paper. Please propose a heading for the odd numbered pages of less than 35 characters. Manuscripts, in triplicate, may be sent to any one of the editors. Please classify according to the scheme of Math. Reviews, Index to Vol. 39. Supply name and address of author to whom proofs should be sent. All other communications should be addressed to the managing editor, or Elaine Barth, University of California, Los Angeles, California, 90024.

50 reprints to each author are provided free for each article, only if page charges have been substantially paid. Additional copies may be obtained at cost in multiples of 50 .

The Pacific Journal of Mathematics is issued monthly as of January 1966. Regular subscription rate: $\$ 84.00$ a year (6 Vols., 12 issues). Special rate: $\$ 42.00$ a year to individual members of supporting institutions.

Subscriptions, orders for numbers issued in the last three calendar years, and changes of address should be sent to Pacific Journal of Mathematics, P.O. Box 969, Carmel Valley, CA 93924, U.S.A. Older back numbers obtainable from Kraus Periodicals Co., Route 100, Millwood, NY 10546.

PUBLISHED BY PACIFIC JOURNAL OF MATHEMATICS, A NON-PROFIT CORPORATION

Printed at Kokusai Bunken Insatsusha (International Academic Printing Co., Ltd.). 8-8, 3-chome, Takadanobaba, Shinjuku-ku, Tokyo 160, Japan.

Copyright (C) 1979 by Pacific Journal of Mathematics Manufactured and first issued in Japan 


\section{Pacific Journal of Mathematics}

\section{Vol. 83, No. $2 \quad$ April, 1979}

Patrick Robert Ahern, On a theorem of Hayman concerning the derivative of a

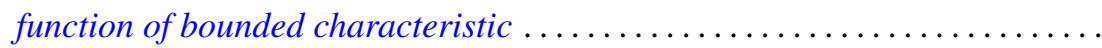

Walter Allegretto, Finiteness of lower spectra of a class of higher order elliptic

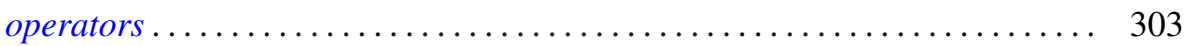

Leonard Asimow, Superharmonic interpolation in subspaces of $C_{c}(X) \ldots \ldots 11$

Steven F. Bellenot, An anti-open mapping theorem for Fréchet spaces . . . . . . . 325

B. J. Day, Locale geometry. . . . . . . . . . . . . . . . . . . . . . . . . . 333

John Erik Fornaess and Steven Krantz, Continuously varying peaking

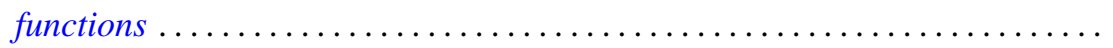

Joseph Leonide Gerver, Long walks in the plane with few collinear points ......

Joseph Leonide Gerver and Lawrence Thom Ramsey, On certain sequences of

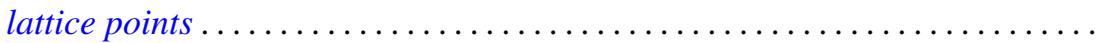

John R. Graef, Yuichi Kitamura, Takaŝi Kusano, Hiroshi Onose and Paul Winton

Spikes, On the nonoscillation of perturbed functional-differential

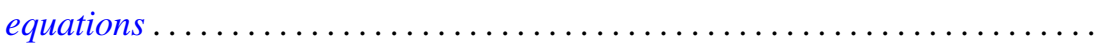

James A. Huckaba and James M. Keller, Annihilation of ideals in commutative

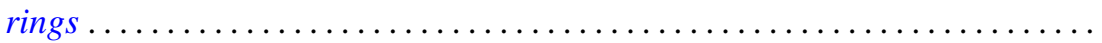

Anzelm Iwanik, Norm attaining operators on Lebesgue spaces . . . . . . . . . . .

Surjit Singh Khurana, Pointwise compactness and measurability . . . .......... 387

Charles Philip Lanski, Commutation with skew elements in rings with

involution.

Hugh Bardeen Maynard, A Radon-Nikodým theorem for finitely additive bounded

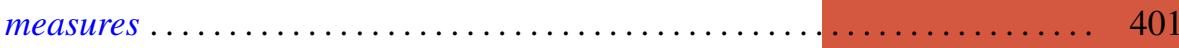

Kevin Mor McCrimmon, Peirce ideals in Jordan triple systems ..

Sam Bernard Nadler, Jr., Joseph E. Quinn and N. Stavrakas, Hyperspaces of compact convex sets.

Ken Nakamula, An explicit formula for the fundamental units of a real pure

sextic number field and its Galois closure ............

Vassili Nestoridis, Inner functions invariant connected components . .

Vladimir I. Oliker, On compact submanifolds with nondegenerate parallel

normal vector fields.

Lex Gerard Oversteegen, Fans and embeddings in the plane.

Shlomo Reisner, On Banach spaces having the property G.L

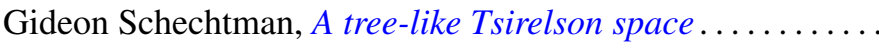

Helga Schirmer, Fix-finite homotopies . . . . . . . . . . . .

Jeffrey D. Vaaler, A geometric inequality with applications to linear forms . .

William Jennings Wickless, $T$ as an $\mathscr{G}$ submodule of $G$.....

Kenneth S. Williams, The class number of $Q(\sqrt{-p})$ modulo 4 , for $p \equiv 3$ (

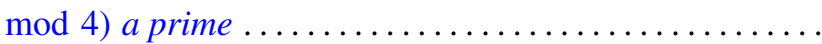

